\title{
Travailler dans l'ESS : aspirations, représentations et dispositions
}

Une étude auprès des adhérents de l'association Ressources solidaires

\section{Working in the social and solidarity economy: Personal goals, representations and attitudes}

\author{
A study of the members of the nonprofit organization \\ Ressources solidaires
}

\section{Fanny Darbus et Matthieu Hély}

Numéro 317, août 2010

URI : https://id.erudit.org/iderudit/1020882ar

DOI : https://doi.org/10.7202/1020882ar

Aller au sommaire du numéro

Éditeur(s)

Association Recma

ISSN

1626-1682 (imprimé)

2261-2599 (numérique)

Découvrir la revue

Citer cet article

Darbus, F. \& Hély, M. (2010). Travailler dans l'ESS : aspirations, représentations et dispositions : une étude auprès des adhérents de l'association Ressources solidaires. Revue internationale de l'économie sociale, (317), 68-86.

https://doi.org/10.7202/1020882ar
Résumé de l'article

A partir d'une étude menée auprès des adhérents d'un site spécialisé dans l'économie sociale et solidaire (ESS) dont il diffuse les offres d'emploi, cet article s'attache à dessiner le portrait type de l'aspirant salarié de l'ESS et plus spécifiquement dans le monde associatif, qui regroupe l'essentiel de l'emploi dans ce champ. Si les premières conclusions de cette étude ne sont donc pas généralisables à l'emploi dans les coopératives et les mutuelles, elles sont riches d'enseignements sur les représentations et les dispositions des actifs souhaitant travailler dans l'ESS. Loin de l'image du cadre supérieur du secteur capitaliste " en quête de sens ", l'étude révèle que le « profil type " est davantage à chercher parmi les jeunes actifs, souvent des femmes fortement diplômées, dont les parents sont salariés de la fonction publique et qui voient se réduire considérablement leurs chances de titularisation dans le service public. 


\section{TRAVAILLER DANS L'ESS: ASPIRATIONS, REPRÉSENTATIONS ET DISPOSITIONS Une étude auprès des adhérents de l'association Ressources solidaires}

* CSE, EHESS. Mél.: fannydarbus @ hotmail.com.

** IDHE Paris ouest-Nanterre. Mél.: matthieu.hely@u-paris10.fr.

\author{
par Fanny Darbus* et Matthieu Hély**
}

A partir d'une étude menée auprès des adhérents d'un site spécialisé dans l'économie sociale et solidaire (ESS) dont il diffuse les offres d'emploi, cet article s'attache à dessiner le portrait type de l'aspirant salarié de l'ESS et plus spécifiquement dans le monde associatif, qui regroupe l'essentiel de l'emploi dans ce champ. Si les premières conclusions de cette étude ne sont donc pas généralisables à l'emploi dans les coopératives et les mutuelles, elles sont riches d'enseignements sur les représentations et les dispositions des actifs soubaitant travailler dans l'ESS. Loin de l'image du cadre supérieur du secteur capitaliste "en quête de sens", l'étude révèle que le "profil type " est davantage à chercher parmi les jeunes actifs, souvent des femmes fortement diplômées, dont les parents sont salariés de la fonction publique et qui voient se réduire considérablement leurs chances de titularisation dans le service public.
(1) Union de syndicats et groupements d'employeurs représentatifs dans l'économie sociale (Usgeres, www.usgeres.fr).

(2) Le syndicat Asso (www. syndicat-asso. fr), dont la première réunion publique s'est tenue le 7 avril 2010, a pour objet la défense des "travailleurs" (au-delà des seuls salariés) du secteur associatif sans distinction sectorielle. Il est affilié à I'union syndicale Solidaires. omme l'ensemble des structures économiques, le monde de l'économie sociale et solidaire (ESS) connait actuellement un profond renouvellement générationnel du fait du départ à la retraite des cohortes d'actifs nés après la Libération. Ce renouvellement prend néanmoins un visage particulier dans cet univers qui, à l'exception de certains secteurs protégés du secteur social et médico-social, n’avait pas connu jusqu'alors de carrières organisées sur le long terme. En effet, les trajectoires biographiques avaient été jusqu'ici plutôt caractérisées par la formation sur le tas et l'autodidaxie. Or, la multiplication des formations de l'enseignement supérieur (tant dans les universités que dans les grandes écoles) orientées vers l'économie sociale et solidaire (Demoustier et Wilson-Courvoisier, 2009) et l'organisation du dialogue social avec la consolidation des institutions de représentation des employeurs, au sein de l'Usgeres ${ }^{(1)}$, et des salariés ${ }^{(2)}$ sont en passe de métamorphoser profondément les pratiques et les acteurs de cet espace en voie de structuration. Faire carrière dans ce secteur n'est plus toujours perçu comme un parcours de marginalisation sur le marché du travail. Certaines organisations adoptent d'ailleurs une rhétorique explicitement managériale et se tournent, dans leurs pratiques de recrutement, vers les élèves des grandes écoles (Groupe SOS, 2009). Travailler dans l'économie sociale et solidaire 
(3) www.lemploi-responsable. com.

(4) www.limesurvey.org. est ainsi devenu une aspiration forte parmi les jeunes diplômés, qui sont nombreux à fréquenter les salons de "l'emploi responsable " organisés à Paris ${ }^{(3)}$ et à l'échelle locale par les institutions de l'ESS. C'est dans ce contexte que nous avons voulu mieux explorer le profil des individus qui recherchaient une activité professionnelle dans l'ESS. Qui sont ces travailleurs? En quoi se différencient-ils du reste de la population active? Se caractérisent-ils par rapport au travail particulier? Quels sont leurs parcours scolaires? Que sont-ils prêts à accepter pour réaliser leur projet? Quelles sont leurs représentations de l'ESS?

A cet effet, nous avons mené une enquête portant sur les adhérents d'un site Internet dédié à l'emploi dans le secteur de l'ESS, Ressources solidaires (www.ressources-solidaires.org; lire l'encadré 1). Sa principale fonction est de proposer des annonces d'emploi à ses adhérents. Un questionnaire a été adressé à cette population par voie électronique. Le logiciel libre Lime Survey ${ }^{(4)}$ a été employé pour administrer et enregistrer les réponses. Une seule diffusion du lien Internet a été réalisée par un message collectif envoyé par le délégué général de Ressources solidaires à environ 4000 adhérents ou anciens adhérents de l'association. Mi-décembre 2009, au terme d'un mois de collecte, 242 personnes avaient répondu au questionnaire. Le nombre de questionnaires entièrement exploitables (renseignés de façon exhaustive) s'élève à 184. C'est donc sur les 184 questionnaires renseignés intégralement que se fonde notre étude. Il convient de préciser que la population choisie et le mode d'administration du questionnaire ont sans doute pour effet de surreprésenter les individus les plus jeunes et familiarisés avec l'usage des nouvelles technologies. Il n'est donc pas surprenant de constater que la majorité de notre échantillon est composé d'individus âgés de 30 ans et moins, d'un niveau de qualification très élevé. Nous ne prétendons donc pas à une quelconque conformité de notre échantillon avec une population des individus " aspirant à travailler dans l'ESS ", qu'il serait par ailleurs difficile de définir

\section{Encadré 1 \\ Travailler dans l'ESS ou travailler dans une association?}

Créé en 2002 par des acteurs de l'économie sociale, le site Ressources solidaires (RS, www. ressources-solidaires.org) compte parmi les premiers à se consacrer à l'économie sociale et solidaire, participant ainsi activement à la reconnaissance du secteur. Lassociation qui le porte a notamment pour objet la promotion des valeurs, des activités et des offres d'emploi provenant spécifiquement des structures de l'ESS. Toutefois, la quasi-totalité des offres concerne le monde associatif, les coopératives et les mutuelles n'abondant que marginalement le fil emploi du site. La présente étude portant exclusivement sur les adhérents de RS, nous maintenons la locution « l'ESS » en sachant qu'au cours de nos analyses elle ne renvoie qu'au monde associatif. 
de façon objective compte tenu de la diversité des trajectoires et des caractéristiques qui la composent ${ }^{(5)}$. Notre enquête permet en revanche d'apporter un éclairage sur les jeunes actifs, sortant de formation initiale (dans la quasi-totalité des cas de l'enseignement supérieur) et ayant pour projet professionnel d'occuper un emploi, durablement ou non, dans l'ESS.

\section{Des aspirations contrastées selon les caractéristiques sociodémographiques}

(5) Si notre enquête ne prétend pas à la représentativité des aspirants à l'emploi dans I'ESS, au vu de la labilité du concept, il n'existe cependant pas à notre connaissance de dispositif méthodologique plus pertinent pour appréhender cette population. Une étude consistant à s'adresser aux responsables de master, licence et titre RNCP qui proposent des formations ESS et qui effectuent un suivi de leurs stagiaires permettrait sans doute d'affiner les résultats. Nous poursuivons pour notre part cette approche exploratoire avec des entretiens auprès des utilisateurs du site Ressources solidaires.

(6) Rappelons que les femmes représentent $68 \%$ des salariés du monde associatif (Tchernonog, 2007).

(7) Voir Céreq, 2008. Ces résultats sont corroborés par les analyses effectuées auprès des praticiens de l'ESS de Midi-Pyrénées étudiés dans le cadre de la thèse menée par Darbus, 2009 (voir en particulier le chapitre 2).
Les participants à l'enquête sont ou ont été adhérents de l'association Ressources solidaires (RS), fondée en 2002 et dont l'objet est de recenser les offres d'employeurs sur le marché de l'emploi auprès des organismes de l'économie sociale et solidaire. A cette fin, elle a constitué une base de données composée des coordonnées et des expériences professionnelles et bénévoles des demandeurs d'emploi dans le secteur.

\section{Des femmes jeunes et diplômées}

Le profil sociodémographique (tableau 1, en page suivante) des personnes interrogées surreprésente très nettement les individus les plus jeunes, puisque près la moitié ont 30 ans ou moins. Cette particularité, liée en grande partie au mode de passation du questionnaire qui favorise les pratiquants des nouvelles technologies, présente l'intérêt d'ajuster la focale sur les jeunes actifs qui aspirent à débuter leur carrière dans l'ESS.

Un fait est ici vérifié: ce sont très largement des femmes qui se destinent à une activité professionnelle au sein de l'ESS. Il accompagne ainsi la tendance historique à l'intégration de la main-d'œuvre féminine au sein du salariat. La majorité des secteurs d'activité qui composent l'ESS recrutent plutôt des femmes ${ }^{(6)}$, notamment la culture, l'action sociale, la santé, l'éducation et la formation. Les organisations de l'ESS attirent, mais également recherchent des jeunes diplômées en communication, en management des ressources humaines et en gestion. Enfin, l'incertitude des carrières dans l'ESS et leur faible attractivité en termes de rémunération peuvent expliquer que notre échantillon se compose seulement pour un tiers d'hommes.

Du point de vue du niveau de formation initiale, la très forte proportion de diplômés du supérieur mérite d'être soulignée. Si bien sûr le mode d'enquête favorise la participation des pratiquants réguliers des outils informatiques, il ne faudrait pas surestimer ce biais lié à l'instrument de collecte de nos données. Une comparaison avec les enquêtes générations menées par le Centre d'études et de recherche sur les emplois et les qualifications (Céreq) fait en effet apparaitre que, sur une génération de jeunes diplômés interrogés trois ans après avoir terminé leur formation initiale dans l'année 2004, 42 \% sont diplômés du supérieur ${ }^{(7)}$.

Cette comparaison se justifie avec notre échantillon, puisque la valeur médiane de la variable de l'année de sortie des études correspond 


\section{Tableau 1}

\section{Les aspirants à l'ESS, profil sociodémographique}

\begin{tabular}{|l|r|r|r|r|}
\hline & Effectif & $\%$ & $\begin{array}{r}\text { Fréquence } \\
\text { cumulée }\end{array}$ & $\begin{array}{r}\text { Pourcentage } \\
\text { cumulé }\end{array}$ \\
\hline Femme & 124 & 67,4 & 124 & 67,4 \\
\hline Homme & 60 & 32,6 & 184 & 100,0 \\
\hline Age & \multicolumn{5}{|l|}{} \\
\hline 30 ans et moins & 88 & 48,6 & 88 & 48,6 \\
\hline Entre 30 et 45 ans & 63 & 34,8 & 151 & 83,4 \\
\hline Plus de 45 ans & 30 & 16,6 & 181 & 100,0 \\
\hline
\end{tabular}

Niveau de formation

\begin{tabular}{|l|r|r|r|r|}
\hline BEP, CAP & 2 & 1,1 & 2 & 1,1 \\
\hline Bac & 9 & 5,1 & 11 & 6,2 \\
\hline Bac + 2 & 14 & 7,9 & 25 & 14,0 \\
\hline Bac + 4 ou maitrise & 12 & 6,7 & 37 & 20,8 \\
\hline $\begin{array}{l}\text { Diplôme d'une école } \\
\text { de commerce ou d'ingénieurs }\end{array}$ & 22 & 12,4 & 59 & 33,2 \\
\hline Doctorat & 6 & 3,4 & 65 & 36,5 \\
\hline Licence & 16 & 9,0 & 81 & 45,5 \\
\hline Master 2 & 97 & 54,5 & 178 & 100,0 \\
\hline
\end{tabular}

Origine géographique

\begin{tabular}{|l|r|r|r|r|}
\hline Franciliens & 19 & 10,7 & 19 & 10,7 \\
\hline Parisiens & 33 & 18,5 & 52 & 29,2 \\
\hline Régions & 126 & 70,8 & 178 & 100,0 \\
\hline
\end{tabular}

Source: enquête en ligne auprès des adhérents de Ressources solidaires, 2009.

(8) Voir le site du Conseil des entreprises, employeurs et groupements de l'économie sociale (Ceges): www.ceges.org. Bien qu'également incomplète, une liste d'une cinquantaine de formations figure en annexe de Draperi, 2009. également à 2004. Or dans notre échantillon, seuls onze individus ne disposent pas d'un diplôme du supérieur (a minima bac +2 ), soit $6 \%$ des répondants. L'ESS se révèle donc particulièrement attractive pour les jeunes diplômés du supérieur. Sans doute faut-il voir ici les effets de l'institutionnalisation des formations universitaires spécialisées dans l'économie sociale. Résultant d'une longue tradition universitaire (notamment à Paris I avec Claude Vienney ou au CNRS puis à l'EPHE avec Henri Desroches), celles-ci se sont en effet multipliées et le Ceges recensait vingt-huit formations labellisées en $2008^{\left({ }^{(8)}\right.}$. Force est de constater que même de grandes écoles de commerce (HEC, Essec...) offrent aujourd'hui à leurs étudiants des spécialisations optionnelles touchant plus ou moins au champ de l'économie sociale. 
(9) Bien que les neuf dixièmes des associations ne soient pas employeurs, les associations emploient au 31 décembre 2006 1,7 million de salariés (sources Insee-Clap, présentées dans CNCress, 2008)
La structuration professionnelle de l'ESS est donc également liée au recrutement d'une main-d'œuvre de plus en plus qualifiée et formée aux pratiques de gestion.

\section{A la recherche d'un emploi ou en poste dans une association}

La situation actuelle des individus ayant participé à l'enquête apporte un éclairage supplémentaire sur la morphologie sociale des travailleurs de l'ESS. La situation modale des femmes (graphique 1) est d'être à la recherche d'un emploi (en particulier pour les femmes âgées de plus de 30 ans). Plus du tiers des répondantes occupent cependant un emploi dans l'ESS et plus précisément dans le secteur associatif, qui est de loin le premier employeur de salariés ${ }^{(9)}$. Les hommes se déclarent un peu moins fréquemment que les femmes à la recherche d'un emploi (cela concerne "seulement » $35 \%$ d'entre eux). Lorsqu'ils ont un emploi, c'est également dans l'ESS. Enfin, une part légèrement plus importante des répondants, relativement aux femmes, occupent un emploi à l'extérieur de l'ESS, dans la fonction publique ou le secteur marchand.

Au-delà des situations déclarées, la principale différence entre hommes et femmes est la tranche d'âge. Plus de la moitié des femmes ont moins d'une trentaine d'années et commencent leur carrière professionnelle. A l'inverse, moins du tiers des hommes sont âgés de 30 ans ou moins,

\section{Graphique 1}

Situation au moment de l'enquête selon l'âge, femmes

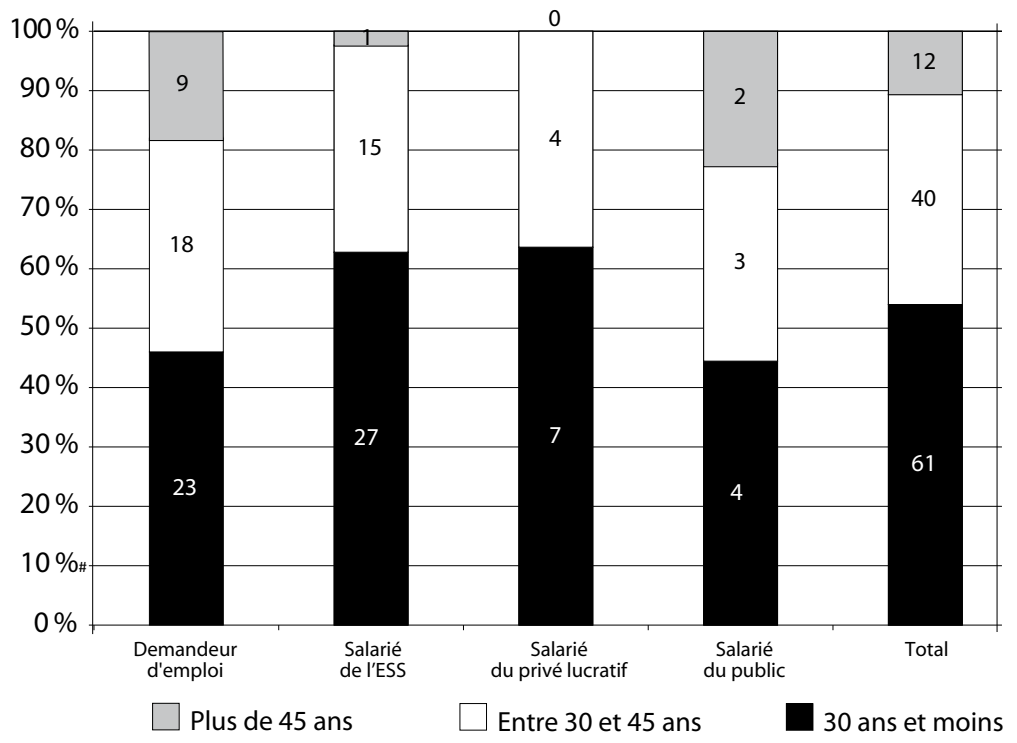

Source: enquête auprès des adhérents de Ressources solidaires, $n=113$. 
(10) Dans le secteur mutualiste et dans quelques grands établissements coopératifs, cependant, les conditions de travail et d'emploi des femmes sont plus favorables que dans le reste de I'ESS. ce qui correspond à une proportion équivalente aux individus âgés de plus de 45 ans. Autrement dit, les hommes - qui, rappelons-le, ne représentent qu'un tiers des répondants - sont plus âgés (l'âge médian est de 33 ans contre 30 ans chez les femmes toutes situations confondues) et plus fréquemment en poste à l'extérieur de l'ESS. En outre, nous avons constaté que la recherche spécifique d'un emploi dans l'ESS était fortement corrélée avec l'âge, puisque cette pratique concerne la moitié des 30 ans et moins contre seulement $25 \%$ des plus de 45 ans. Ces résultats convergent pour conclure que l'emploi salarié dans l'ESS suscite plus d'aspirations professionnelles chez les jeunes femmes que chez les hommes en milieu, voire en fin de carrière professionnelle. Dans la population des demandeurs d'emploi, l'examen des âges médians des femmes et des hommes conforte l'idée d'une trajectoire de reconversion vers l'ESS: la moitié des hommes se déclarant à la recherche d'un emploi sont âgés de 38 ans et plus, contre 31 ans et moins chez les chômeuses. Par ailleurs, les individus masculins ayant participé à l'enquête apparaissent davantage en difficulté sur le marché du travail, puisqu'ils sont systématiquement plus nombreux à avoir déclaré des séquences répétées de chômage pouvant aller jusqu’à dix-huit mois consécutifs. Ces données sont confortées par le fait que $35 \%$ d'entre eux déclarent avoir déjà fait l'expérience d'au moins un licenciement, contre $22 \%$ des femmes.

\section{Une trajectoire professionnelle discontinue}

La population enquêtée apparaît donc fortement segmentée selon l'âge, le sexe (graphique 2, en page suivante) et la trajectoire sur le marché du travail. Première catégorie d'individus, les jeunes femmes diplômées du supérieur qui débutent leur vie professionnelle et aspirent à la poursuivre dans l'ESS. En effet, les entreprises de l'ESS (les coopératives mises à part, [CNCress 2008]) accompagnent certainement davantage que les autres secteurs, compte tenu de l'importance de la main-d'œuvre féminine, le processus de féminisation de la population active et du marché du travail. Mais dans le même temps, cette féminisation prend moins les traits de l'émancipation que ceux de l'exposition plus fréquente à la précarité de l'emploi (contrats courts et temps partiel) et à la discrimination salariale. Et sur ce point, il a été démontré, dans le cadre d'une analyse «toutes choses égales par ailleurs", que les "entreprises associatives " se caractérisent par un salariat nettement moins protecteur que les entreprises du secteur marchand (Hély, 2009). Le fait que les femmes soient plus nombreuses à travailler dans l'ESS ne veut donc pas dire que les modalités de leur mise au travail diffèrent significativement des pratiques dominantes sur le marché du travail ${ }^{(10)}$.

Deuxième catégorie de travailleurs, les hommes en milieu, voire en fin de carrière, dont la trajectoire professionnelle s'écarte de l'idéal de référence hérité de la société salariale et fondé sur la carrière stable avec progression linéaire au sein de la même organisation. Dans une perspective de reconversion professionnelle, l'ESS peut représenter une voie possible 


\section{Graphique 2 \\ Sexe des enquêtés selon la tranche d'âge}

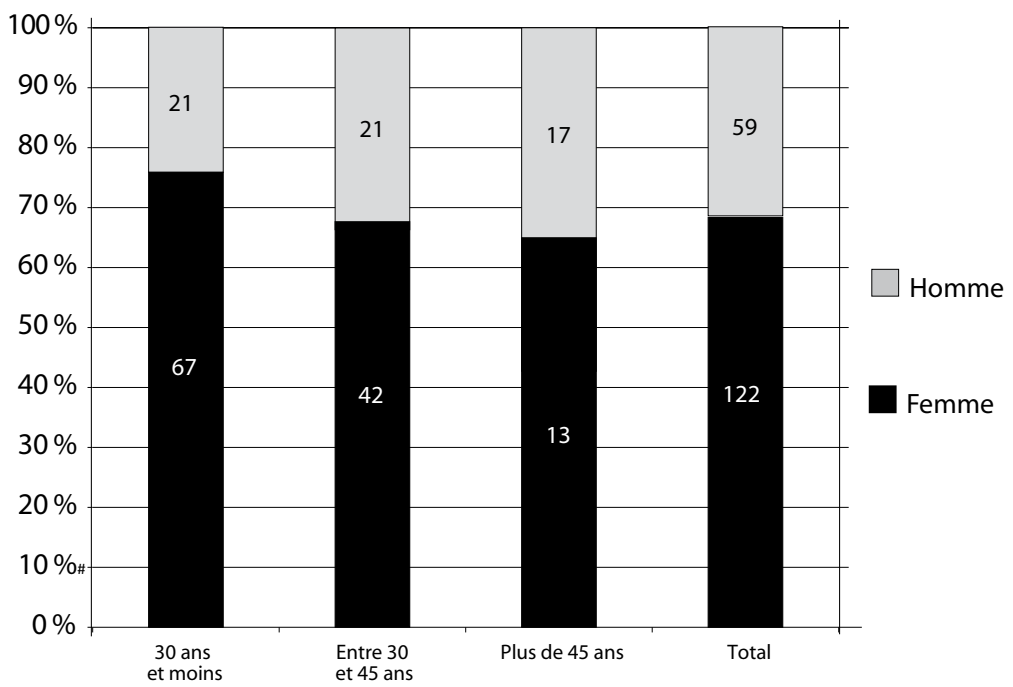

Source: enquête auprès des adhérents de Ressources solidaires, $n=181$.

(11) « Mode de vie des personnes selon l'âge et le sexe ", téléchargeable sur www.insee.fr. de carrière, mais certainement pas exclusive. Une partie de l'échantillon se caractérise en effet par un rapport à l'emploi assez discontinu: 20\% ont connu au moins une fois dix-huit mois de chômage minimum. Ce sont en particulier les plus âgés, les plus de 45 ans qui ont connu le plus le chômage, et cela à la plus grande fréquence: $50 \%$ des plus de 45 ans ont connu au moins une fois plus de dix-huit mois de chômage. Sans surprise, un chômage de courte durée est observé chez les jeunes en sortie de formation initiale.

\section{Des femmes sans enfant}

Du point de vue des structures familiales, les jeunes femmes ayant participé à l'enquête se singularisent par une forte proportion de personnes déclarant vivre seules sans enfant (tableau 2, en page suivante). A titre de comparaison, cette catégorie particulière ne représentait que $8,2 \%$ des femmes âgées de 30 à 44 ans selon le recensement de l'Insee en $1999^{(11)}$, alors qu'elle représente près d'un tiers de notre échantillon pour cette modalité. Selon le recensement de l'Insee, les deux tiers des femmes de cette tranche d'âge vivaient en couple avec enfant(s). Elles sont très nettement minoritaires dans notre enquête. Les femmes qui aspirent à travailler dans l'ESS se trouvent dans des configurations familiales particulières, puisque la majorité d'entre elles n'ont pas d'enfant. S'agit-il d'une particularité de notre échantillon? Deux hypothèses se posent alors: 


\section{Tableau 2}

Statut conjugal et familial selon l'âge

\begin{tabular}{|c|c|c|c|c|c|}
\hline & $\begin{array}{c}\text { En couple } \\
\text { avec enfant(s) }\end{array}$ & $\begin{array}{r}\text { En couple } \\
\text { sans enfant }\end{array}$ & $\begin{array}{c}\text { Seul(e) } \\
\text { avec enfant(s) }\end{array}$ & $\begin{array}{c}\text { Seul(e) } \\
\text { (ou hébergé[e] } \\
\text { par ses parents) } \\
\text { sans enfant }\end{array}$ & Total \\
\hline \multicolumn{6}{|c|}{ Femmes $(n=113)$} \\
\hline $\begin{array}{l}\text { Moins } \\
\text { de } 30 \text { ans }\end{array}$ & $12,9 \%$ & $41,9 \%$ & $0,0 \%$ & $45,2 \%$ & $100,0 \%$ \\
\hline $\begin{array}{l}\text { Entre } 30 \\
\text { et } 45 \text { ans }\end{array}$ & $37,5 \%$ & 20,0 & $12,5 \%$ & $30,0 \%$ & $100,0 \%$ \\
\hline \begin{tabular}{|l|} 
Plus \\
de 45 ans
\end{tabular} & $23,1 \%$ & $0,0 \%$ & $46,2 \%$ & $30,8 \%$ & $100,0 \%$ \\
\hline \begin{tabular}{|l|} 
Total \\
\end{tabular} & $22,6 \%$ & $29,6 \%$ & $9,6 \%$ & $38,3 \%$ & $100,0 \%$ \\
\hline $\begin{array}{l}\text { Moins } \\
\text { de } 30 \text { ans }\end{array}$ & $10,0 \%$ & $60,0 \%$ & $0,0 \%$ & $30,0 \%$ & $100,0 \%$ \\
\hline \multicolumn{6}{|c|}{ Hommes $(n=57)$} \\
\hline $\begin{array}{l}\text { Entre } 30 \\
\text { et } 45 \text { ans } \\
\end{array}$ & $38,1 \%$ & $38,1 \%$ & $4,8 \%$ & $19,0 \%$ & $100,0 \%$ \\
\hline $\begin{array}{l}\text { Plus } \\
\text { de } 45 \text { ans }\end{array}$ & $50,0 \%$ & $12,5 \%$ & $25,0 \%$ & $12,5 \%$ & $100,0 \%$ \\
\hline Total & $31,6 \%$ & $38,6 \%$ & $8,8 \%$ & $21,1 \%$ & $100,0 \%$ \\
\hline
\end{tabular}

Source: enquête auprès des adhérents de Ressources solidaires, 2009.

soit cette spécificité correspond à un projet familial différé (rappelons que l'âge médian des femmes est de 30 ans); soit il peut s'agir d'une hiérarchisation des priorités où la vie professionnelle est privilégiée par rapport à la fondation d'un foyer, hypothèse qu'étayent de nombreuses études (Meron, Widmer, 2002; Baudelot, Establet, 2000). Les variables relatives au rapport au travail révèlent en effet des attentes importantes à l'égard de l'épanouissement professionnel « toutes choses égales par ailleurs ».

Du point de vue des hommes, nous constatons une plus grande conformité des structures familiales à l'égard des données nationales recueillies par l'Insee. La majorité des individus interrogés déclarent vivre en couple. Résumons-nous. D'après notre enquête, le profil des travailleurs de l'ESS, en poste ou à la recherche d'un emploi, se caractérise par une forte 
féminisation, un niveau de diplômé très élevé et des configurations familiales particulières chez les femmes, un faible nombre d'entre elles ayant des enfants.

\section{Les représentations du travail dans l'économie sociale et solidaire à l'épreuve des pratiques}

(12) Le troisième secteur en France, "Travail et société " pour le compte de la CEE, juin 1978 .
Depuis les années 70, les entreprises de l'économie sociale invoquent l'utopie du " travail autrement » pour se différencier du rapport au travail caractéristique de l'économie capitaliste. Le rapport rédigé par Jacques Delors et Jocelyne Gaudin pour la Communauté européenne apparait ainsi emblématique des aspirations orientées vers cet idéal: «Parce qu’il permet de travailler autrement, le troisième secteur sécrète des possibilités importantes dans deux directions essentielles pour une politique du travail: davantage de flexibilité dans les horaires de travail ou dans la pondération des différentes activités, l'accès à des formes d'autogestion (self-management) permettant d'accéder à une participation plus approfondie et à des formes originales de démocratie industrielle ${ }^{(12)}$. " Les représentations positives développées dans ce rapport sont révélatrices de l'enchantement des rapports de production dans l'économie sociale et de la dénégation de ces derniers, notamment, par les travaux académiques de nombreux chercheurs en sciences sociales (Hély, 2009). Le mythe de la « réconciliation de l'économique et du social ", régulièrement invoqué par les institutions de l'économie sociale pour se différencier des rapports de production capitalistes fondés sur l'antagonisme des classes, donne à penser qu'une autre forme d'organisation du travail est possible dans ces entreprises qui entendent « replacer l'homme au cœur de leur projet ".

Lorsque l'on examine les réponses des adhérents de Ressources solidaires, force est de constater que peu d'entre eux s'illusionnent sur le montant des rémunérations et sur la stabilité des emplois offerts par l'économie sociale et solidaire.

\section{Une plus grande autonomie, mais des conditions salariales estimées moins bonnes}

Plus de $80 \%$ des répondants à notre enquête désapprouvent les propositions selon lesquelles les rémunérations dans l'ESS seraient équivalentes à celles du secteur marchand, et les emplois, plus stables (graphique 3, en page suivante). Notons que la majorité d'entre eux consultent régulièrement les offres d'emploi mises en ligne sur le site Internet et qu'ils sont donc très bien informés des conditions de travail dans l'ESS. Ce sont les questions sur l'organisation du travail qui suscitent le plus de clivages. L'item selon lequel l'ambiance de travail serait meilleure dans l'ESS recueille ainsi une majorité de réponses positives. Sans doute faut-il voir ici l'effet des valeurs collectives incarnées par l'ESS, où la solidarité et la réciprocité sont centrales. L'autonomie des salariés dans l'organisation 


\section{Graphique 3}

Les représentations du travail dans I'ESS par les adhérents de RS

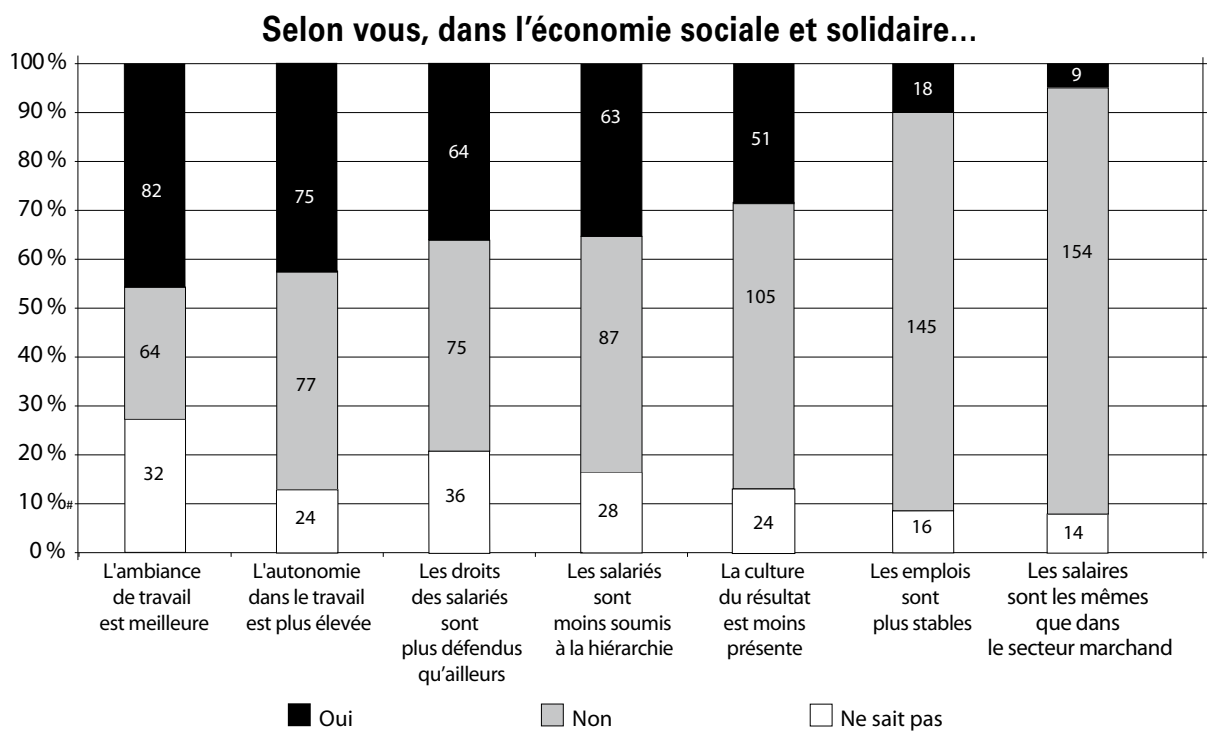

Source: enquête auprès des adhérents de Ressources solidaires, 2009.

est la deuxième modalité où la fréquence des réponses positives est la plus élevée. A noter que la situation des répondants influe nettement sur la structure des réponses, puisque les salariés de l'ESS sont ceux qui obtiennent le taux de réponses positives le plus important (dix points au-dessus de la moyenne). Sans doute est-ce l'organisation du travail par projets, dans le cadre de tâches faiblement prescrites et nécessitant un niveau de qualification élevé, qui permet d'expliquer ce résultat. Pour autant, autonomie dans le travail ne signifie pas absence de contrainte hiérarchique, puisque, pour la majorité des répondants, les salariés de l'ESS ne sont pas moins soumis à la subordination que dans d'autres secteurs. En revanche, l'esprit d'initiative et la culture du projet laissent plus de libertés. L'injonction àl'évaluation des projets mis en œuvre par les organisations de l'ESS pour l'obtention de partenariats avec les institutions est une donnée qui n'échappe pas à la conscience des répondants. La majorité d'entre eux se déclarent ainsi conscients que la " culture du résultat " n’est pas moins présente dans l'ESS qu'ailleurs. En revanche, la proposition selon laquelle les droits des salariés seraient mieux défendus dans l'ESS que dans d'autres secteurs est celle qui recueille le plus grand nombre d'abstentions. Il est frappant de constater que seuls 18 individus sur 69 salariés dans l'ESS (graphique 4, en page suivante) approuvent l'idée selon laquelle leurs organisations seraient plus protectrices de leurs droits. C'est la fréquence la plus faible par rapport à la structure des réponses sur l'ensemble des répondants. 
(13) Voir Bernard Gorce, "L'association Emmaüs secouée par un conflit social ", La Croix, 8 mars 2010.
La faible application des dispositions légales du Code du travail et de réglementations des conditions de travail (horaires, rémunérations, congés, etc.) par l'intermédiaire des conventions collectives constitue sans aucun doute l'une des raisons majeures de ce constat. Le domaine des relations professionnelles est en effet un espace où les organisations de l'ESS pensent être en mesure de faire valoir leurs spécificités. Mais appartenir à l'ESS ne suffit au développement de pratiques vertueuses ou socialement responsables. En effet, les spécificités juridiques des organismes n'exemptent pas les salariés qui y travaillent de différentes formes de domination ou d'exploitation, comme l'illustre par exemple le récent conflit au sein de l'association Emmaüs ${ }^{(13)}$.

\section{Les dispositions de l'adhésion à l'économie sociale et solidaire}

Au total, 53,8 \% des adhérents de Ressources solidaires déclarent qu’au moins un de leurs parents est ou a été agent de la fonction publique.

Graphique 4

Structure des réponses à la proposition "Les salariés seraient mieux défendus dans l'économie sociale et solidaire qu'ailleurs ", selon le statut du répondant

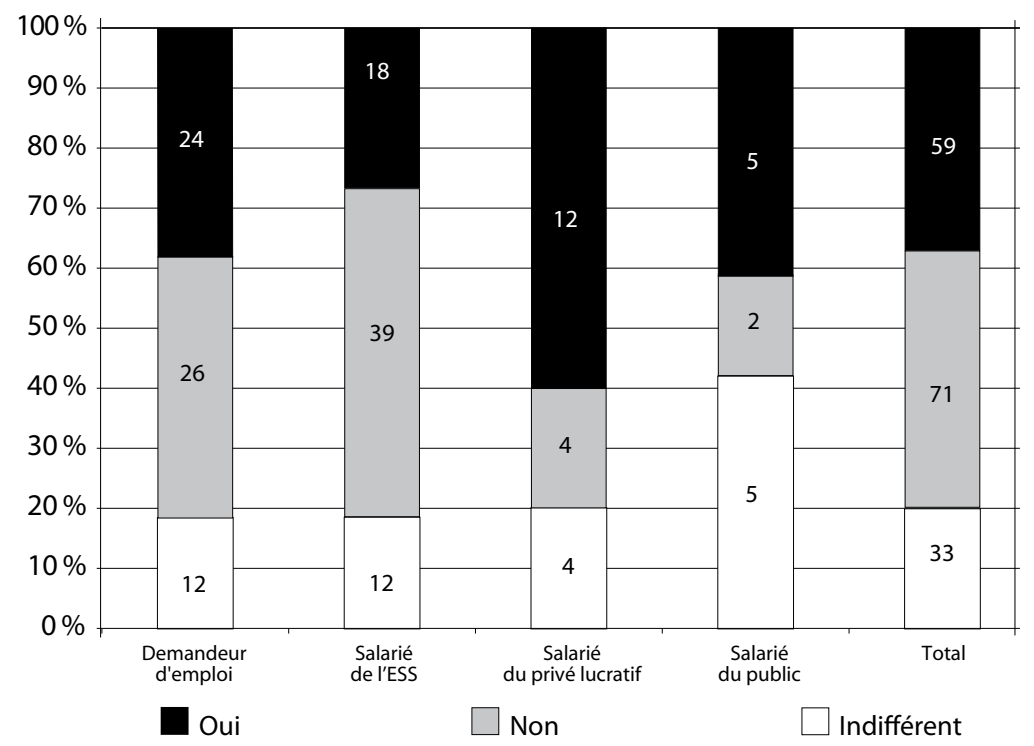

Source: enquête auprès des adhérents de Ressources solidaires, 2009. 
(14) Des études plus particulièrement universitaires. Les diplômés d'école de commerce ou d'ingénieurs sont en effet nettement sous-représentés parmi les enfants de fonctionnaires.
Il s'agit d'une proportion très élevée par rapport à l'ensemble de la population active. En effet, selon les données de l'enquête "Formation, qualification professionnelle " de l'Insee, cette situation caractérisait à peine un tiers des actifs occupés en 2003. Cette surreprésentation des enfants de fonctionnaires, déjà confirmée par d'autres sources, conforte donc la thèse d'une homologie de position entre les agents de la fonction publique, dotés de dispositions à servir l'intérêt général souvent acquises par la socialisation familiale et l'institution scolaire, et les travailleurs de l'ESS, plus particulièrement les salariés du secteur associatif (Hély, 2009). Faut-il parler d'un ethos du service public pour caractériser l'adhésion aux valeurs de solidarité et d'égalité, longtemps incarnées par les institutions républicaines? Face au déclin de la légitimité des services publics, soumis aux dogmes du new public management et à la diminution importante des effectifs de personnel de la fonction publique, les organisations de l'économie sociale et solidaire apparaissent en effet comme des acteurs de plus en plus essentiels dans la mise en œuvre de l'action et des politiques publiques.

\section{Du service public à l'ESS?}

Lorsque l'on étudie plus finement la part des individus dont l'un des parents au moins est ou a été salarié de la fonction publique, il est frappant de constater que la fréquence la plus élevée $(60 \%)$ se situe dans la catégorie des 30 ans et moins. L'hypothèse d'un destin professionnel contrarié pour de jeunes actifs ayant investi dans les études supérieures ${ }^{(14)}$ pour accroître leurs chances d'entrer dans la fonction publique est plutôt confortée par ces résultats. En outre, plus de $25 \%$ des individus ont déclaré avoir passé un ou plusieurs concours de la fonction publique. Ces prédispositions pour l'emploi public, acquises par la socialisation familiale et l'institution scolaire, doivent en effet s'accommoder d'une évolution très profonde des chances d'accès aux postes de titulaire. On n'insistera jamais assez sur la situation exceptionnelle qui caractérise notre époque: l'emploi public, qui n'avait jamais cessé sa progression dans la population active depuis deux siècles, connaît pour la première fois une inversion orientée vers une diminution durable. Or, cette situation intervient alors que le nombre d'enfants de fonctionnaires n'a jamais été aussi élevé. On sait que le souhait d'être fonctionnaire a toujours été très élevé, notamment en période de crise du marché du travail, où la fonction publique représente des valeurs de sécurité et de stabilité fortement prisées (De Singly, Thélot, 1988). Mais on sait également, depuis les analyses d'Alain Darbel et de Dominique Schnapper menées dans les années 60, que la fonction publique se caractérise par un taux d'hérédité professionnelle parmi les plus élevés de la population active occupée: un fils de fonctionnaire a deux fois plus de chances qu'un autre de devenir lui-même fonctionnaire (Darbel, Schnapper, 1969). Le nombre de fils et de filles de fonctionnaire n'ayant jamais été aussi élevé qu'aujourd'hui, nombre d'aspirations à servir la collectivité devront s'épanouir au-delà de la fonction publique. 
Dans la fonction publique d'Etat, la mise en œuvre du principe de nonremplacement d'un départ à la retraite sur deux conduit à une diminution régulière depuis le début des années 2000. Dans la fonction publique territoriale, où les emplois de non-titulaire ont toujours été plus importants, l'embauche sur contrat s'est imposée comme le principal mode de recrutement au détriment de la titularisation dans le cadre du statut (Biland, 2009). Il faut sans doute voir ici les conséquences de la transposition du droit communautaire par la loi du 26 juillet 2005 qui consacre la possibilité de recourir au contrat à durée indéterminée.

\section{Ethos entrepreneurial}

La proximité des activités de l'ESS avec les missions d'intérêt général dévolues aux administrations publiques vient souligner les affinités sociologiques entre les travailleurs de l'ESS et les agents de la fonction publique: une surreprésentation des femmes, un niveau de diplôme élevé, l'acquisition d'un ethos du service public, la famille et un rapport au travail probablement moins orienté vers la réussite matérielle que dans d'autres secteurs de l'économie. Cependant, ces affinités contrastent avec les conditions objectives de ces deux catégories. Statuts d'emploi précaire et salaires dévalorisés par les normes sociales de l'ESS, où il est de bon ton de revendiquer désintéressement et don de soi au nom de "l'esprit associatif ", caractérisent en effet la situation de la majorité des travailleurs de ce secteur. Tout se passe donc comme si une force de travail était en train de se constituer, sociologiquement proche du groupe social des fonctionnaires, mais socialisée dans des conditions objectives qui sont inversement symétriques aux normes du statut protecteur de la fonction publique. $\mathrm{Ou}$ pour le dire autrement, nous assisterions, au-delà des discours incantatoires sur l'idéal du "travail autrement" qu'incarnerait l'ESS, à la naissance d'une nouvelle fraction du salariat remplissant les missions du public dans les conditions du privé. Précisons d'emblée que cette thèse, si elle est confirmée par les résultats de l'enquête, ne doit pas être généralisée à l'ensemble de la population étudiée. En effet, si une majorité des individus concentrent des caractéristiques spécifiques qui les rapprochent du secteur public, ce n'est bien sûr pas le cas de la totalité. Il convient de ne pas subsumer l'hétérogénéité intrinsèque des expériences de l'ESS derrière une thèse univoque. L'ethos entrepreneurial, acquis par la fréquentation des écoles de commerce, caractérise également une part importante des répondants. Il faut donc se garder d'une radicalisation de la thèse qui consisterait à dénoncer une privatisation masquée de l'emploi public au nom de la promotion de l'ESS. Les trajectoires individuelles et la diversité des expériences vécues ne permettent pas de tenir telle analyse.

\section{Accepter de gagner moins?}

Pour comprendre ce que peuvent accepter les adhérents de Ressources solidaires pour travailler dans l'ESS, nous leur avons soumis une série de propositions que nous leur demandions d'approuver ou non. Parmi 
Tableau 3

Pour travailler dans l'économie sociale et solidaire, êtes-vous prêt à consentir à une rémunération plus faible qu'ailleurs?

\begin{tabular}{|l|r|r|r|r|r|}
\hline Homme & \multicolumn{4}{|r|}{ Statut conjugal et familial } & \\
\hline & $\begin{array}{r}\text { En couple } \\
\text { avec enfant(s) }\end{array}$ & $\begin{array}{r}\text { En couple } \\
\text { sans enfant }\end{array}$ & $\begin{array}{r}\text { Seul } \\
\text { avec enfant(s) }\end{array}$ & $\begin{array}{r}\text { Seul } \\
\text { sans enfant }\end{array}$ & Total \\
\hline Oui & $68,4 \%$ & $95,2 \%$ & $100,0 \%$ & $75,0 \%$ & $82,1 \%$ \\
\hline Non & $31,6 \%$ & $4,8 \%$ & $0,0 \%$ & $25,0 \%$ & $17,9 \%$ \\
\hline Total & $100,0 \%$ & $100,0 \%$ & $100,0 \%$ & $100,0 \%$ & $100,0 \%$ \\
\hline
\end{tabular}

Source: enquête auprès des adhérents de Ressources solidaires, hommes uniquement, $n=56$.

\begin{tabular}{|l|r|r|r|r|r|}
\hline Femme & \multicolumn{3}{|r|}{ Statut conjugal et familial } & \\
\hline & $\begin{array}{r}\text { En couple } \\
\text { avec enfant(s) }\end{array}$ & $\begin{array}{r}\text { En couple } \\
\text { sans enfant }\end{array}$ & $\begin{array}{r}\text { Seule } \\
\text { avec enfant(s) }\end{array}$ & $\begin{array}{r}\text { Seule } \\
\text { sans enfant }\end{array}$ & Total \\
\hline Oui & $84,0 \%$ & $73,5 \%$ & $45,5 \%$ & $82,2 \%$ & $76,5 \%$ \\
\hline Non & $16,0 \%$ & $26,5 \%$ & $54,5 \%$ & $17,8 \%$ & $23,5 \%$ \\
\hline Total & $100,0 \%$ & $100,0 \%$ & $100,0 \%$ & $100,0 \%$ & $100,0 \%$ \\
\hline
\end{tabular}

Source: enquête auprès des adhérents de Ressources solidaires, femmes uniquement, $n=115$.

celles-ci, le consentement à une rémunération plus faible était évoqué. Près de $80 \%$ des individus (tableau 3) se déclarent prêts à accepter une rémunération plus faible pour travailler dans l'ESS. Cette réponse est cohérente avec les représentations du secteur. Les adhérents sont en effet conscients de l'existence d'un différentiel de rémunération significatif avec le secteur marchand. Ils l'ont donc intériorisé et l'acceptent très nettement. Si l'on prend en compte l'influence des structures familiales, il est frappant de constater une grande différence, à situation équivalente, entre les attitudes des hommes et celles des femmes. Les hommes qui se déclarent les plus récalcitrants à l'hypothèse de gagner moins en travaillant dans l'ESS sont dans la situation familiale modale du couple avec enfant(s). A l'inverse, les femmes vivant seules avec enfant(s) déclarent refuser en majorité de gagner moins pour travailler dans l'ESS. C'est d'ailleurs la seule catégorie, tous sexes confondus, qui se distingue par une proportion plus importante d'individus refusant l'hypothèse de la perte de rémunération. Si les adhérents de Ressources solidaires ne se font pas d'illusion sur l'impact salarial du travail dans l'ESS et acceptent très largement de gagner moins pour réaliser leur idéal, le différentiel de rémunération entre ESS et secteur marchand apparaît comme anormal pour près des trois quarts d'entre eux. Là encore, l'étude des structures familiales est éclairante. 
Ce différentiel est jugé « normal » pour près d'un tiers des femmes vivant seules sans enfant ou hébergées par leurs parents. Ce taux est quasiment équivalent pour les hommes dans la même situation familiale. Labsence d'anticipation des contraintes conjugales et familiales favorise ainsi l'acceptation de l'inégalité salariale.

A quel niveau se situe le degré d'acceptation des adhérents de Ressources solidaires pour travailler dans l'ESS? De nouveau, la situation familiale est un puissant élément d'explication. Si la majorité des femmes se déclarent plutôt réticentes à l'hypothèse d'un recrutement dans le cadre d'une mesure d'insertion pour travailler dans l'ESS, les trois quarts de celles qui vivent seules avec enfant(s) se déclarent prêtes à l'accepter (tableau 4). Le taux d'acceptation pour les hommes est de seulement $20 \%$ (avec des effectifs faibles).

\section{Pour des horaires flexibles en contrat temporaire}

Sur la question de la flexibilité des horaires, les attitudes des femmes et celles des hommes se distinguent radicalement: plus de $80 \%$ des hommes se déclarent prêts à l'accepter, contre environ $40 \%$ des femmes (tableau 5 , en page suivante). Pour les femmes, la vie en couple a un effet clivant: le fait de vivre à deux favorise l'acceptation, alors que vivre seule favorise une attitude de refus. Sur cette dimension, les femmes prennent davantage en compte leur situation familiale dans l'acceptation des contraintes professionnelles. Il reste cependant à savoir si les femmes n'ayant pas d'enfant privilégient leur épanouissement personnel plutôt que leur carrière.

Enfin, la flexibilité de l'emploi est davantage acceptée par les femmes que par les hommes. Les femmes seules sont davantage enclines à l'accepter soit par pure contrainte économique (enfant[s] à charge), soit parce qu'elles sont en début de vie professionnelle et prêtes à de nombreux compromis pour débuter leur carrière.

\section{Tableau 4}

Pour travailler dans l'économie sociale et solidaire, êtes-vous prête à être embauchée dans le cadre de mesures d'insertion?

\begin{tabular}{|l|r|r|r|r|r|}
\hline & \multicolumn{3}{|c|}{ Statut conjugal et familial } \\
\hline \\
$\begin{array}{r}\text { En couple } \\
\text { ave enfant(s) }\end{array}$ & $\begin{array}{r}\text { En couple } \\
\text { sans enfant }\end{array}$ & $\begin{array}{r}\text { Seule } \\
\text { avec enfant(s) }\end{array}$ & $\begin{array}{r}\text { Seule } \\
\text { sans enfant }\end{array}$ & Total \\
\hline Ne sait pas & $20,8 \%$ & $26,5 \%$ & $11,1 \%$ & $18,2 \%$ & $20,7 \%$ \\
\hline Non & $54,2 \%$ & $50,0 \%$ & $11,1 \%$ & $36,4 \%$ & $42,3 \%$ \\
\hline Oui & $25,0 \%$ & $23,5 \%$ & $77,8 \%$ & $45,5 \%$ & $36,9 \%$ \\
\hline Total & $100,0 \%$ & $100,0 \%$ & $100,0 \%$ & $100,0 \%$ & $100,0 \%$ \\
\hline
\end{tabular}

Source: enquête auprès des adhérents de Ressources solidaires, femmes uniquement, $n=111$. 


\section{Tableau 5}

Pour travailler dans l'économie sociale et solidaire, êtes-vous prête
à accepter des horaires flexibles?

\begin{tabular}{|l|r|r|r|r|r|}
\hline & \multicolumn{3}{|r|}{ Statut conjugal et familial } & \\
\hline & $\begin{array}{r}\text { En couple } \\
\text { avec enfant(s) }\end{array}$ & $\begin{array}{r}\text { En couple } \\
\text { sans enfant }\end{array}$ & $\begin{array}{r}\text { Seule } \\
\text { avec enfant(s) }\end{array}$ & $\begin{array}{r}\text { Seule } \\
\text { sans enfant }\end{array}$ & Total \\
\hline Oui & $72,0 \%$ & $75,8 \%$ & $8,3 \%$ & $7,0 \%$ & $41,6 \%$ \\
\hline Non & $28,0 \%$ & $24,2 \%$ & $91,7 \%$ & $93,0 \%$ & $58,4 \%$ \\
\hline Total & $100,0 \%$ & $100,0 \%$ & $100,0 \%$ & $100,0 \%$ & $100,0 \%$ \\
\hline
\end{tabular}

Source: enquête auprès des adhérents de Ressources solidaires, femmes uniquement, $n=115$.

(15) On note que $60 \%$ des salariés "hors ESS ", dans I'entreprise classique et la fonction publique, déclarent avoir des objectifs de travail non atteignables.
L'hypothèse qui demande à être développée et à laquelle les résultats de l'enquête ne permettent pas de répondre de façon satisfaisante est la possibilité que l'économie sociale et solidaire constitue un espace où les opportunités de carrière sont plus ouvertes que dans d'autres secteurs pour les femmes (Tchernonog, 2005; Tabariès, Tchernonog, 2007).

\section{Une recherche exclusive dans I'ESS?}

Seuls $45 \%$ de l'ensemble des adhérents recherchent exclusivement un emploi dans l'ESS. Ils sont $56 \%$ à être dans ce cas parmi les salariés associatifs, ce qui tend à indiquer que, globalement, ceux qui appartiennent déjà au secteur souhaitent y rester. On notera que la probabilité de rechercher spécifiquement un emploi dans l'ESS est fortement dépendante de l'âge. C'est le cas d'un jeune actif de 30 ans et moins sur deux, contre à peine un quart des plus de 45 ans. Les données confirment donc l'idée d'un fort effet générationnel lié à la structuration récente de l'ESS comme espace professionnel où "faire carrière " devient une aspiration légitime (sans toutefois que cette expression signifie que ce soit au sein de la même organisation et au même poste). Les plus âgés, notamment s'ils travaillent dans des entreprises lucratives ou dans le secteur public, aspirent surtout à quitter leur poste, sans viser de secteur d'arrivée particulier ${ }^{(15)}$. Tout se passe donc comme si l'ESS constituait un secteur auquel prétendent de manière éventuelle un grand nombre de travailleurs développant ou ayant développé un rapport malheureux à l'emploi. La population salariée qui aspire à rejoindre l'ESS se caractérise en effet par un rapport à l'emploi relativement heurté: le nombre de ruptures d'emploi, comme celui d'employeurs, est élevé: $62 \%$ des salariés ont déjà démissionné, la plupart d'entre eux étant plutôt âgés de plus de 45 ans. Par ailleurs, $83 \%$ des enquêtés en poste au moment de l'enquête se sentent surqualifiés et plus de $60 \%$ d'entre eux se déclarent mal, voire très mal payés. Ce sont plutôt les individus les plus âgés qui déplorent ce phénomène, pendant que 
(16) Certains pourraient s'étonner de ce que ce taux n'atteigne pas $100 \%$ du fait de l'adhésion à l'association Ressources solidaires. L'échantillon comporte d'anciens adhérents plus à jour de leur cotisation et notre question posait la condition de " membre à titre bénévole ", suggérant donc une implication active dans l'organisation, au-delà de la simple cotisation. Quoi qu'il en soit, ce taux de participation bénévole aux activités du monde associatif est plus important que celui observé dans la population générale. Les entretiens semi-directifs qui vont être réalisés à la suite de cette enquête quantitative devront explorer de façon plus approfondie cette dimension. Ces engagements peuvent-ils être également le vecteur d'opportunités professionnelles, notamment pour les personnes en situation de recherche d'emploi? les plus jeunes semblent faire preuve d'un certain stoïcisme. On note l'existence d'une population de précaires de la fonction publique, puisque, parmi ceux qui ont déjà travaillé pour l'administration, déclarent avoir été employés par plus de douze employeurs $50 \%$ des plus de 45 ans qui ont déjà travaillé pour une administration publique et $75 \%$ pour une entreprise privée lucrative.

"Chargé de mission » constitue très largement le poste le plus recherché par les adhérents du site d'emploi Ressources solidaires. Cet intitulé évasif est évoqué dans un cas sur deux, surtout par les moins de 30 ans. Les plus âgés évoquent quant à eux, pour leur recherche, des postes de "cadre " ou de "direction". C'est principalement dans le secteur de l'accompagnement à l'emploi et de l'insertion, secondairement dans le secteur développement durable, du sanitaire social et de l'humanitaire, et enfin dans celui de la culture que recherchent les candidats à l'ESS. Secteur de l'ESS mis à part, c'est au sein du service public que les adhérents au site étudiés souhaiteraient travailler. En dépit de la variété des formes d'emploi flexible que propose l'ESS, la grande majorité de ceux qui veulent s'y consacrer cherchent des postes stables (CDI) et à temps plein. Cela va donc à l'encontre du discours du "travailleur nomade ", organisant son " portefeuille de compétences » et cultivant son employabilité au gré des projets auxquels il participe, revendiqué par de nombreux promoteurs $\mathrm{du}$ secteur (Menger, 2009; Auer, Gazier, 2006).

\section{Des aspirants par ailleurs " militants "?}

Parmi les individus de l'échantillon, plus de six sur dix se déclarent membres d'une association à titre bénévole ${ }^{(16)}$, et ce taux atteint $85 \%$ si l'on prend en compte celles et ceux qui déclarent avoir déjà été membres, mais ne le sont plus au moment de l'enquête. Enfin, le fort engagement associatif n'est pas corrélé avec d'autres formes de participation politique: $10 \%$ sont syndiqués (on note d'ailleurs que la majorité des syndiqués sont des travailleurs associatifs, le plus souvent dans un syndical affilié à la CFDT), tandis qu'une minorité, $7 \%$, déclarent une adhésion militante au sein d'un parti politique, généralement de gauche modéré (Parti socialiste ou Les Verts).

\section{Conclusion}

(17) "L'économie sociale et solidaire: "Une quête de sens" ", in Lemonde.fr (9 décembre 2009), d'où sont également tirées les citations de cette conclusion.
Au-delà du discours convenu sur la "quête de sens ${ }^{(17)}$ " qui caractérisait les individus aspirant à travailler dans l'ESS, force est de constater que c'est d'abord et avant tout la situation de recherche d'emploi qui conditionne l'orientation vers ce secteur. La situation modale des adhérents (ou ex-adhérents) de Ressources solidaires est d'être au chômage. Le "profil type " du cadre supérieur qui a "parfaitement réussi sa carrière " et qui, à 40 ans, est " en quête de sens " et se demande pourquoi "il a passé sa vie à vendre des tonnes et des tonnes de yaourts, sans passer beaucoup de temps 
(18) Pour mémoire, entre 1948 et 1972 , le nombre d'agents publics en France était passé de 2 millions à 3 millions et demi. auprès de sa famille, pour un salaire honnête » est assez nettement éloigné de ce que l'enquête permet d'observer. S'il y a bel et bien un " profil type ", il est plutôt à rechercher parmi les jeunes actifs fortement diplômés dont les parents sont ou ont été dans la fonction publique et qui, confrontés à un effondrement inédit des chances d'accéder à un poste de titulaire dans une administration d'Etat ou une collectivité territoriale ${ }^{(18)}$, réajustent leurs aspirations initiales en direction des entreprises de l'ESS, ces dernières, à la différence du secteur marchand, étant sans doute les mieux à même de garantir une intégration professionnelle sans contrarier trop fortement les dispositions acquises par la socialisation familiale et scolaire. 


\section{Bibliographie}

Auer P., Gazier B., 2006, L'introuvable sécurité de l'emploi, Flammarion.

Baudelot Ch., Establet R., 2000, Avoir 30 ans en 1968 et en 1998, Seuil.

Céreq, 2008, Quand l'école est finie, premiers pas dans la vie active de la génération 2004, www.cereq.fr.

Biland E., 2009, "L'emploi public local et la réforme de l'Etat ", www.laviedesidees.fr.

CNCress, 2008, Panorama de l'économie sociale et solidaire, www.cncress.ort.

Darbel A., Schnapper D., 1969, Les agents du système administratif, Paris, La Haye, Mouton. Darbus F., 2009, " Pratiques et praticiens de l'économie sociale et solidaire, 2000-2007: contribution à la sociologie des croyances économiques ", EHESS, Paris, juin.

De Singly F., Thélot C., 1988, Gens du privé, gens du public: la grande différence, Dunod.

Demoustier D., Wilson-Courvoisier S., 2009, "L'enseignement initial de l'économie sociale et solidaire: un enjeu stratégique ", Recma, no 311 .
Draperi J.-F., 2009, L'année de l'économie sociale et solidaire, une alternative à redécouvrir en temps de crise, Dunod.

Groupe SOS, 2009, Conversations solidaires, Editions Rue de l'Echiquier.

Hély M., 2009, Les métamorphoses du monde associatif, Puf.

Menger P.-M., 2009, Le travail créateur: s'accomplir dans l'incertain, Gallimard-Seuil. Meron M., Widmer I., 2002, "Les femmes au chômage retardent l'arrivée du premier enfant ", Population, vol. 57, p. 327-357.

Tabariès M., Tchernonog V., 2007, « La montée des femmes dirigeantes dans les associations: évolutions structurelles, évolutions sociétales ", Recma, n 303.

Tchernonog V., 2005, "Les femmes dans les associations: la non-mixité des bureaux, reflet de centres d'intérêt différents ou modalité d'accession aux responsabilités pour les femmes?", Recma, n' 297.

Tchernonog V., 2007, Le paysage associatif français, Dalloz, "Juris Associations ". 